\title{
LIE GROUPS AND LIE ALGEBRAS IN ROBOTICS
}

\author{
J.M. Selig \\ South Bank University \\ London SE1 OAA, U.K. \\ seligjm@sbu.ac.uk
}

\begin{abstract}
In this lecture the group of rigid body motions is introduced via its representation on standard three dimensional Euclidian space. The relevance for robotics is that the links of a robot are usually modelled as rigid bodies. Moreover the payload of a robot is also usually a rigid body and hence much of robotics is concerned with understanding rigid transformations and sequences of these transformations. Chasles's theorem is presented, that is: a general rigid body motion is a screw motion, a rotation about a line in space followed by a translation along the line.

The lower Reuleaux pairs are introduced. These are essentially surfaces which are invariant under some subgroup of rigid body motions. Such a surface can be the matting surface for a mechanical joint. In this way the basic mechanical joints used in robots can be classified. These surfaces turn out to have other applications in robotics. In robot gripping they are exactly the surfaces that cannot be immobilised using frictionless fingers. In robot vision the symmetries of these surfaces are motions which cannot be detected.

Next Lie algebras are introduced. The 1-parameter rigid motions about a joint are considered. The correspondence between elements of the Lie algebra and 1-degree-of-freedom joints is given. The exponential map from the Lie algebra to the group is defined and used to describe the forward kinematics of a serial robot using the product of exponentials formula. The Rodrigues formula for the exponential map is derived using a set of mutually annihilating idempotents.

The derivative of the exponential map is explored. In particular the Jacobian matrix for the forward kinematics is derived. More general results are also derived and these are used to show how the inverse kinematics problem can be cast as a set of ordinary differential equations.
\end{abstract}


Keywords: Clifford algebra, Lie algebras, Lie groups, rigid body motions, robotics.

\section{Introduction-Rigid Body Motions}

A large amount of robotics is concerned with moving rigid bodies around in space. A robot here is usually an industrial robot arm as in figure 1, however most of the material presented here applies to parallel manipulators and closed loop mechanisms. There are also some applications to mobile robots and robot vision.

It is usual to think of the components that make up the links of the robot as rigid and also the payload or tool is often a rigid body. Hence, an important problem in robotics is to keep track of these rigid bodies.

It is well known that any rigid transformation can be composed from a rotation, a translation and a reflection. Physical machines cannot perform reflections and so we should really speak of proper rigid transformations, excluding the reflections. The proper rigid transformations can be represented using $4 \times 4$ matrices. These can be written in partitioned form as

$$
A=\left(\begin{array}{cc}
R & \mathbf{t} \\
0 & 1
\end{array}\right)
$$

where $R$ is a $3 \times 3$ rotation matrix and $\mathbf{t}$ is a translation vector.

The action of these matrices on points in space is given by,

$$
\left(\begin{array}{c}
\mathbf{p}^{\prime} \\
1
\end{array}\right)=\left(\begin{array}{ll}
R & \mathbf{t} \\
0 & 1
\end{array}\right)\left(\begin{array}{l}
\mathbf{p} \\
1
\end{array}\right)=\left(\begin{array}{c}
R \mathbf{p}+\mathbf{t} \\
1
\end{array}\right)
$$

where $\mathbf{p}$ and $\mathbf{p}^{\prime}$ are the original and transformed position vectors of a point.

There are two ways we can use these matrices to describe the position and orientation of a rigid body. If we use an active point of view we can agree on a standard 'home' position for the body, any subsequent position and orientation of the body is described by the rigid transformation which moves the home position of the body to its current position and orientation.

In the passive viewpoint a coordinate frame is fixed in the body, now the position and orientation of the body is given by the coordinate transform which expresses the coordinates of points in the current frame in terms of those in the home frame. For historical reasons, this passive viewpoint seems to be preferred in robotics even though the active viewpoint is often simpler.

These two viewpoints are related quite simply. The transformation given by the passive view is the inverse of the active transformation. To see this assume that the new frame has its origin at a point with position 


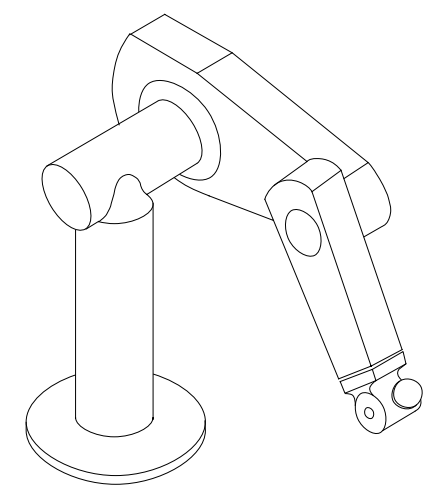

Figure 1. A six-joint industrial robot arm.

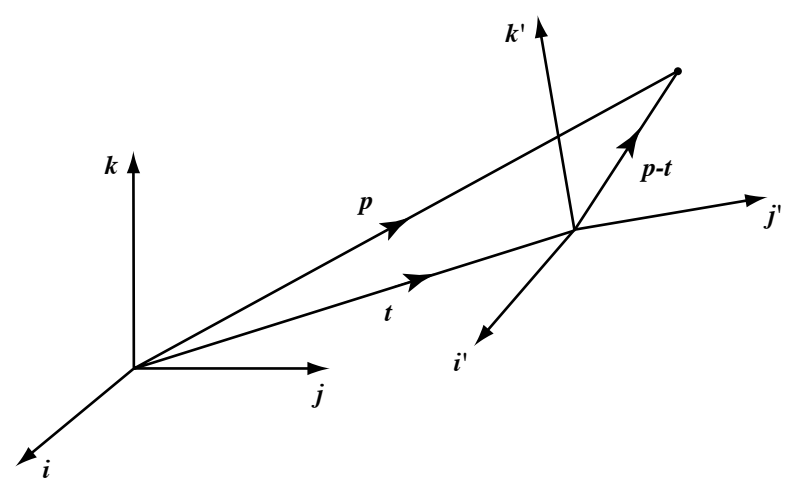

Figure 2. A vector referred to two coordinate frames. 
vector $\mathbf{t}$ with respect to the original frame. Now an arbitrary point $\mathbf{p}$ in the original frame has position vector $\mathbf{p}^{\prime}=\mathbf{p}-\mathbf{t}$ with respect to the new frame, see figure 2. In general the orientation of the new frame will be different to that of the original, assume it is given by a rotation $R$. That is, the basis vectors of the new frame $\mathbf{i}^{\prime}, \mathbf{j}^{\prime}$ and $\mathbf{k}^{\prime}$, are given by,

$$
\mathbf{i}^{\prime}=R \mathbf{i}, \quad \mathbf{j}^{\prime}=R \mathbf{j}, \quad \mathbf{k}^{\prime}=R \mathbf{k}
$$

If we write the position vector of the point as

$$
\mathbf{p}=x \mathbf{i}+y \mathbf{j}+z \mathbf{k}
$$

in the original frame, then in the new frame the point will have coordinates,

$$
\mathbf{p}^{\prime}=x^{\prime} \mathbf{i}^{\prime}+y^{\prime} \mathbf{j}^{\prime}+z^{\prime} \mathbf{k}^{\prime}
$$

where $x^{\prime}=\mathbf{p}^{\prime} \cdot \mathbf{i}^{\prime}=(\mathbf{p}-\mathbf{t})^{T} R \mathbf{i}$ and so forth. Hence the new coordinates can be written in terms of the old as

$$
\left(\begin{array}{l}
x^{\prime} \\
y^{\prime} \\
z^{\prime}
\end{array}\right)=R^{T}\left(\begin{array}{l}
x \\
y \\
z
\end{array}\right)-R^{T} \mathbf{t}
$$

Compare this with the inverse of a general rigid transformation which is given by,

$$
\left(\begin{array}{cc}
R & \mathbf{t} \\
0 & 1
\end{array}\right)^{-1}=\left(\begin{array}{cc}
R^{T} & -R^{T} \mathbf{t} \\
0 & 1
\end{array}\right)
$$

The space of all possible rigid body transformations is an example of a Lie group. So we are led naturally to study Lie groups.

\section{Lie Groups}

In general, groups can be thought of as sets of symmetry operations. Originally these were symmetries of algebraic equations. We will be interested in symmetries of geometric figures here.

The definition of a group is the abstraction of the properties of symmetry operations. There are four axioms. So formally a group is a set $G$ with a binary operation, usually written as juxtaposition.

- The group must be closed under its binary operation.

- The operation must be associative.

- The group must have a unique identity element.

- Every element of the group must have a unique inverse. 
Notice that sets of square matrices can be groups quite easily, the group product is modelled by matrix multiplication, which is associative of course. The identity element of the group is represented by the identity matrix. The inverse of a group element is represented by the inverse matrix, so it is important that the matrices in the set are all non-singular.

Lie groups have to satisfy the following additional axioms.

- The set of group elements $G$ must form a differentiable manifold.

- The group operation must be a differentiable map. As a map the group operation sends $G \times G \longrightarrow G$. On pairs of group elements $g_{1}, g_{2} \in G$ the map has the effect $\left(g_{1}, g_{2}\right) \mapsto g_{1} g_{2}$.

- The map from a group element to its inverse must be a differentiable mapping. On an element $g \in G$ this map has the effect, $g \mapsto g^{-1}$.

A differentiable manifold is essentially a space on which we can do calculus. Locally it looks like $\mathbb{R}^{n}$ but globally it could be quite different. It is usual to think of such a manifold as patched together from pieces of $\mathbb{R}^{n}$. The standard example is a sphere, which can be constructed from two patches, one covering the northern hemisphere and the other the southern hemisphere. The patches can also be thought of as local coordinate systems for the manifold, in general manifolds do not have global coordinate systems.

The set of non-singular $n \times n$ matrices is an example of such a Lie group. The underlying manifold of the group is simply an open set in $\mathbb{R}^{n^{2}}$, that is $\mathbb{R}^{n^{2}}$ with the closed set of determinant zero matrices deleted. The other axioms are clearly satisfied, matrix multiplication is clearly differentiable and so is the inversion map. This group is usually denoted $G L(n)$ for the general linear group of order $n$.

Subspaces of this group can also be groups. A good example for us is the rotation matrices. These are $3 \times 3$ matrices which also satisfy,

$$
R^{T} R=I, \quad \text { and } \quad \operatorname{det}(R)=1 .
$$

These equations define a non-singular algebraic variety in $G L(3)$. It is possible to show that such a subspace is a differentiable manifold. In this case the group manifold is 3-dimensional projective space $\mathbb{P R}^{3}$. This space can be thought of as the space of lines through the origin in $\mathbb{R}^{4}$ or alternatively as the 3 -dimensional sphere with anti-podal points identified. The group is usually denoted $S O(3)$, the special orthogonal group of order three. 
Next we look in detail at the Lie group that we are most concerned with; the group of rigid body transformations.

\section{Finite Screw Motions}

In robotics the group of rigid transformations is called $S E(3)$, it is supposed to denote the special Euclidian group. It is the semi-direct product of $S O(3)$, the rotations about the origin, with $\mathbb{R}^{3}$, the translations.

$$
S E(3)=S O(3) \rtimes \mathbb{R}^{3}
$$

In a direct product the factors would not interact. The semi-direct product indicates that the rotations act on the translations. This can be easily seen from the $4 \times 4$ matrices, if we multiply two such matrices we get,

$$
\left(\begin{array}{cc}
R_{2} & \mathbf{t}_{2} \\
0 & 1
\end{array}\right)\left(\begin{array}{cc}
R_{1} & \mathbf{t}_{1} \\
0 & 1
\end{array}\right)=\left(\begin{array}{cc}
R_{2} R_{1} & R_{2} \mathbf{t}_{1}+\mathbf{t}_{2} \\
0 & 1
\end{array}\right)
$$

Notice how the first translation vector is rotated before it is added to the second one.

The group manifold for a semi-direct product of groups is simply the Cartesian product of the manifolds of the factors. So the group manifold for $S E(3)$ is simply the Cartesian product of $\mathbb{P R}^{3}$ with $\mathbb{R}^{3}$. Notice that this is therefore also the configuration manifold for a rigid body, each position and orientation of the body corresponds to a group element and vice versa.

We end this section with a sketch of Chasles's theorem. This, originally geometric, theorem dates back to the 1830s, well before Lie's work on 'continuous groups'. The theorem states that every rigid transformation (with the exception of pure translations) is a finite screw motion. That is, a rotation about a line together with a translation along the line, see figure 3 .

A finite screw motion about a line through the origin has the form

$$
A(\theta)=\left(\begin{array}{cc}
R & \frac{\theta p}{2 \pi} \hat{\mathbf{x}} \\
0 & 1
\end{array}\right)
$$

where $\hat{\mathbf{x}}$ is a unit vector along the axis of the rotation, $\theta$ the angle of rotation, and $p$ the pitch of the motion. Since the axis of rotation is $\hat{\mathbf{x}}$ we also expect that $R \hat{\mathbf{x}}=\hat{\mathbf{x}}$, in other words $\hat{\mathbf{x}}$ is an eigenvector of $R$ with unit eigenvalue.

In general, if the line doesn't pass through the origin the transformation can be found by conjugation. Suppose $\mathbf{u}$ is a point on the line, then we can translate $\mathbf{u}$ back to the origin, perform the screw motion above 


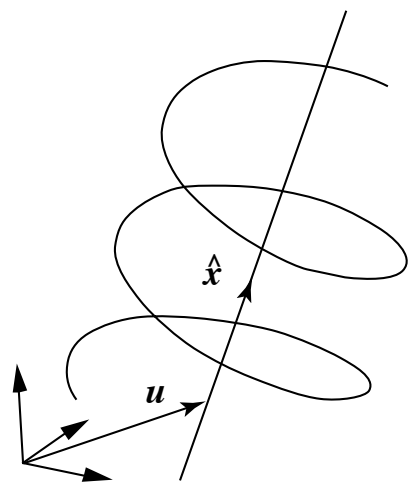

Figure 3. A finite screw motion.

and finally translate the origin back to $\mathbf{u}$. This gives

$$
\left(\begin{array}{cc}
I & \mathbf{u} \\
0 & 1
\end{array}\right)\left(\begin{array}{cc}
R & \frac{\theta p}{2 \pi} \hat{\mathbf{x}} \\
0 & 1
\end{array}\right)\left(\begin{array}{cc}
I & \mathbf{u} \\
0 & 1
\end{array}\right)=\left(\begin{array}{cc}
R & \frac{\theta p}{2 \pi} \hat{\mathbf{x}}+(I-R) \mathbf{u} \\
0 & 1
\end{array}\right)
$$

Now Chasles's theorem amounts to the following: Given an arbitrary rigid transformation it can always be put in the above form. That is, we have to solve,

$$
\left(\begin{array}{cc}
R & \mathbf{t} \\
0 & 1
\end{array}\right)=\left(\begin{array}{cc}
R & \frac{\theta p}{2 \pi} \hat{\mathbf{x}}+(I-R) \mathbf{u} \\
0 & 1
\end{array}\right)
$$

for $p$ and $\mathbf{u}$ given $\mathbf{t}$ and $R$. Assuming we can find $\theta$ and $\hat{\mathbf{x}}$ from $R$ then it is not too difficult to see that

$$
\frac{\theta p}{2 \pi}=\hat{\mathbf{x}} \cdot \mathbf{t}
$$

This gives the pitch $p$. Now we have a system of linear equations for $\mathbf{u}$,

$$
(I-R) \mathbf{u}=\frac{\theta p}{2 \pi} \hat{\mathbf{x}}-\mathbf{t} .
$$

These equations are singular, but the kernel of $(I-R)$ is clearly $\hat{\mathbf{x}}$ and so the equations are consistent and it will be possible to find a solution for $\mathbf{u}$ up to addition of an arbitrary multiple of $\hat{\mathbf{x}}$. In practice, it would be sensible to require that $\mathbf{u}$ be perpendicular to $\hat{\mathbf{x}}$. If the transformation is a pure translation then $R=I$ and the above fails, this is the only case where $\mathbf{u}$ cannot be found. Pure rotations correspond to pitch zero, $p=0$. Screw motions and pure translations are often taken to be screw motions with infinite pitch. 
Notice that if we have two screw motions about the same line with the same pitch then these transformations commute. It is only necessary to check this for lines through the origin, the conjugation can be used to extend this easily to the general case. So for example we have

$$
\left(\begin{array}{cc}
R\left(\theta_{2}\right) & \frac{\theta_{2} p}{2 \pi} \hat{\mathbf{x}} \\
0 & 1
\end{array}\right)\left(\begin{array}{cc}
R\left(\theta_{1}\right) & \frac{\theta_{1} p}{2 \pi} \hat{\mathbf{x}} \\
0 & 1
\end{array}\right)=\left(\begin{array}{cc}
R\left(\theta_{1}+\theta_{2}\right) & \frac{\left(\theta_{1}+\theta_{2}\right) p}{2 \pi} \hat{\mathbf{x}} \\
0 & 1
\end{array}\right)
$$

Remember, the rotations here are about the same axis. From this it can be seen that the set of all screw motions about the same line with the same pitch constitutes a group. That is, the set is closed under the group operation. These are the one-parameter subgroups of $S E(3)$.

\section{Mechanical Joints}

At the end of the nineteenth century Franz Reuleaux described what he called "lower pairs" [7]. These were pairs of surfaces which can move relative to each other while remaining in surface contact. He took these to be idealisations for the most basic of mechanical joints. He found six possibilities, see figure 4 .

It is possible to give a simple group theoretic proof that these are the only possibilities. The key observation is that these surfaces must be invariant under some subgroup of $S E(3)$. The subgroup represents the symmetries of the surface. For a joint, the subgroup will give the possible relative motions between the sides of the joint.

To find these surfaces, consider the surfaces invariant under oneparameter (1-dimensional) subgroups.

- The pitch zero subgroups correspond to rotations about a line. A surface invariant under such a subgroup is just a surface of rotation.

- Infinite pitch subgroups correspond to translations in a fixed direction, so any surface of translation is invariant under such a subgroup.

- The subgroups with finite, non-zero pitch have helicoidal surfaces as invariants. These give the first three lower Reuleaux pairs.

To find more Reuleaux pairs consider how the subgroups can be combined to form larger subgroups. Surfaces invariant with respect to these larger subgroups must have more than one of the above properties.

- So the cylinder is a surface of rotation and a surface of translation (and a helicoidal surface for any pitch). 


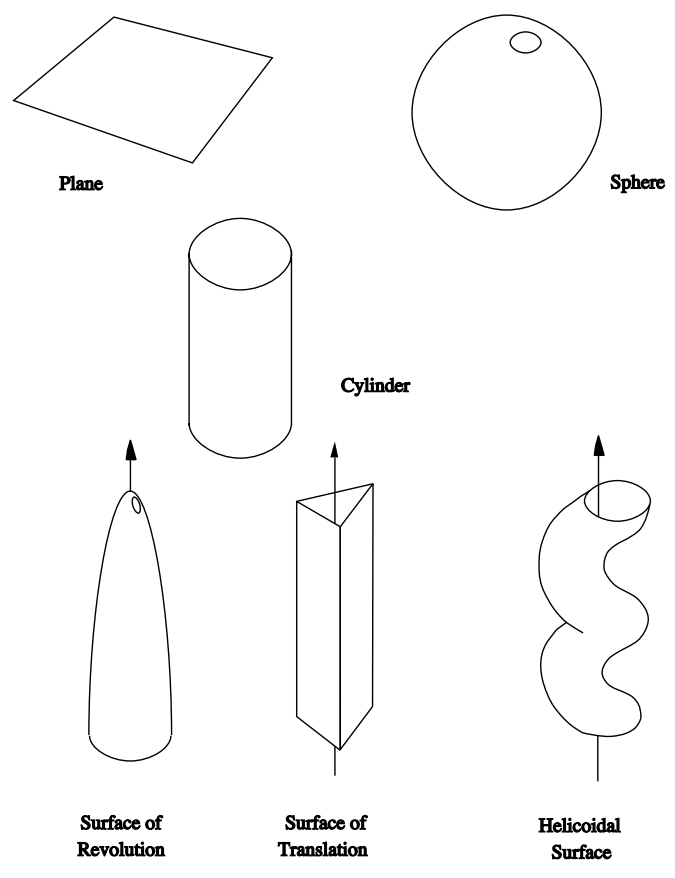

Figure 4. The six lower Reuleaux pairs.

- A sphere is invariant with respect to the subgroup of rotations in space $S O(3)$. That is, the sphere is a surface of rotation about any of its diameters.

- A plane with respect to $S E(2)$ the group of rigid transformations in the plane.

With a little care, it is possible to show that these are the only possibilities - Reuleaux found them all!

In mechanical engineering, the joints corresponding to lower Reuleaux pairs have special names. Simple hinge joints corresponding to the rotational pair are known as revolute joints. Sliding joints corresponding to the translational pair are called prismatic joints and the joints corresponding to the helicoidal pair are helical joints or sometimes screw joints. A ball-and-socket joint is more properly called a spherical joint and the last two pairs form cylindric and planar joints.

Practical robots may have any of these joints, but it is most common to actuate revolute or prismatic joints. Revolute joints can be accurately and easily controlled with electric motors. When larger forces are required hydraulic rams can be used as actuated prismatic joints. 
However, for a hydraulic ram the length of travel is hard to control accurately.

It is possible to find all the subgroups of $S E(3)$. The zero-dimensional subgroups are the point groups, which are subgroups of the rotations only, and the 230 space groups familiar from crystallography. For the higher dimensional subgroups see Hervé [4].

\section{Invisible Motion and Gripping}

The lower Reuleaux pairs turn up in other places in robotics.

Consider trying to constrain an object by placing frictionless fingers on the object's surface. The fingers can only exert forces normal to the surface of the object. It is known that there are some surfaces which cannot be completely constrained by any number of frictionless fingers. These surfaces are precisely the lower Reuleaux pairs.

It is straightforward to see that a lower pair is "ungrippable," the symmetry will move the surface such that the velocity at any point on the surface is perpendicular to the contact normal. Hence, these motions cannot be constrained.

It is a little harder to show that any 'ungrippable' surface must have a symmetry. Essentially, the space of normal lines to the surface are considered as six-dimensional force-torque vectors called wrenches. For an 'ungrippable' surface the normal do not span the six-dimension vector space of wrenches. Hence, there will be a dual six-dimensional vector which annihilates all normal lines, this vector will generate the symmetries. It is an element of the Lie algebra, see below.

In robot vision a common problem is to reconstruct the motion of objects given two or more successive images of the scene. Flow fields are constructed from differences in image intensity. However, it is well known that certain motions of objects cannot be detected in this way, Horn gives the example of a sphere rotating about any diameter, [5].

It is clear that motions of all Reuleaux pairs are undetectable in this way. It is also clear that these will be the only undetectable rigid body motions if we discount discrete symmetries of regular polyhedra exactly synchronised with the succession of images. For example, we would not expect to see the motion of a tetrahedron if it executes a symmetry between the images we capture. On the other hand we are not really limited to rigid motions here. There may be other motions which are invisible to such a system, but this would probably depend very much on the lighting conditions. For example, consider the motion of a sphere again, this time the sphere is moving away from the observer and at the same time is expanding at such a rate that the apparent contour in the 
image remains constant. This would be detectable under point lighting but there might be lighting conditions under which a move like this is undetectable.

\section{Forward Kinematics}

From the above it is clear that motion about a 1-degree-of-freedom joint can be parameterised by a one-parameter subgroups.

The general case is a helical joint where the corresponding 1-parameter subgroup can be written

$$
A(\theta)=\left(\begin{array}{cc}
R & \frac{\theta p}{2 \pi} \hat{\mathbf{x}}+(I-R) \mathbf{u} \\
0 & 1
\end{array}\right)
$$

where $\hat{\mathbf{x}}$ is the direction of the joint axis, $p$ is the pitch of the joint and $\mathbf{u}$ the position vector of a point on the joint axis. Revolute joints are most common in practical machines, these correspond to subgroups of pitch zero,

$$
A(\theta)=\left(\begin{array}{cc}
R & (I-R) \mathbf{u} \\
0 & 1
\end{array}\right)
$$

Sometimes prismatic joints are used, the corresponding 1-parameter subgroup for such a joint has the form,

$$
A(\theta)=\left(\begin{array}{cc}
I & \theta \hat{\mathbf{t}} \\
0 & 1
\end{array}\right)
$$

where $\hat{\mathbf{t}}$ is the direction of the joint. Notice in each of the cases above, the identity element is given when the parameter is zero.

For a serial robot, such as the on illustrated in figure 1, it is important to find the transformation undergone by the end-effector. This problem is usually known as the forward kinematics of the robot. Let us fix a standard or home position for the robot. In the home configuration all the joint variables will be taken to be zero. Now, to move the robot to a position specified by a set of joint variables $\boldsymbol{\theta}^{T}=\left(\theta_{1}, \theta_{2}, \theta_{3}, \theta_{4}, \theta_{5}, \theta_{6}\right)$ we can perform the motions about the joints in turn beginning with the distal joint $\theta_{6}$; the one furthest from the base of the robot. The motion of the end-effector about this joint will be given by the matrix $A_{6}\left(\theta_{6}\right)$. Next we move the fifth joint, the axis of this joint has not be affected by the motion about the last joint and so the effect on the tool is just $A_{5}\left(\theta_{5}\right)$. In this way we can work our way down the arm and deduce the overall transformation on the end-effector or tool to be,

$$
K(\boldsymbol{\theta})=A_{1}\left(\theta_{1}\right) A_{2}\left(\theta_{2}\right) A_{3}\left(\theta_{3}\right) A_{4}\left(\theta_{4}\right) A_{5}\left(\theta_{5}\right) A_{6}\left(\theta_{6}\right)
$$


This expression represents the active transformation undergone by the robot's end-effector relative to the home configuration. It is straightforward to compute the 'A' matrices if we know the position of the robot's joint axes in the home configuration.

In the robotics literature it is also common to use tool-frame coordinates, and to give the forward kinematics in terms of this frame. Specifically, the tool-frame is a coordinate system fixed to the robot's endeffector. We seek the passive, coordinate transformation which converts coordinates in a frame fixed in the base link of the robot to coordinates in the tool frame. Suppose that in the robot's home position the active transformation from the base link frame to the tool frame is given by the matrix $B$. The overall transformation between the base link frame and the tool frame in an arbitrary configuration of the robot will be given by the transformation, $B$ to the tool frame in the home position, followed by the transformation of the end-effector itself. That is $K(\boldsymbol{\theta}) B$. This is still an active transformation, to convert it to a coordinate transformation, as we saw above in section 1, we simply invert the matrix. Hence, the kinematics in terms of tool-frame coordinates is given by,

$$
(K(\boldsymbol{\theta}) B)^{-1}=B^{-1} A_{6}^{-1}\left(\theta_{6}\right) A_{5}^{-1}\left(\theta_{5}\right) A_{4}^{-1}\left(\theta_{4}\right) A_{3}^{-1}\left(\theta_{3}\right) A_{2}^{-1}\left(\theta_{2}\right) A_{1}^{-1}\left(\theta_{1}\right)
$$

\section{Lie Algebra}

In 1900 Ball published his influential treatise on "the theory of screws" [1]. With the benefit of hindsight it is clear that Ball's screws were simply elements of the Lie algebra of $S E(3)$. More precisely, the twists correspond to the element of the Lie algebra, the screws are elements of the projective space formed from the Lie algebra. This distinctions will not be used in the following.

The theory of Lie groups and their Lie algebras was developed some years later by, Lie, Klein, Killing and Cartan among others. So now we are in a position to take advantage of the Lie theoretic viewpoint and update Ball's original screw theory.

The Lie algebra of a Lie group can be defined in several different ways. Historically, Lie algebra elements were thought of as infinitesimal group elements. Here we can think of the Lie algebra of a group as the tangent space at the identity element. To find the Lie algebra elements we take a curve in the group and find the derivative at the identity.

We can use use the $4 \times 4$ representation of $S E(3)$ to find its Lie algebra. As we saw above finite screw motion can be written,

$$
A(\theta)=\left(\begin{array}{cc}
R & \frac{\theta p}{2 \pi} \hat{\mathbf{x}}+(I-R) \mathbf{u} \\
0 & 1
\end{array}\right)
$$


where, $R$ is a rotation through angle $\theta$ about the axis $\hat{\mathbf{x}}$, and $\mathbf{u}$ is a point on the axis. This defines a sequence of rigid transformations parameterised by $\theta$. Moreover, when $\theta=0$ we get the identity transformation.

So taking the derivative at $\theta=0$ gives a typical element of the Lie algebra,

$$
S=\left(\begin{array}{cc}
\Omega & \frac{\omega p}{2 \pi} \hat{\mathbf{x}}-\Omega \mathbf{u} \\
0 & 0
\end{array}\right)
$$

Here $\Omega=d R(0) / d \theta$ is a $3 \times 3$ anti-symmetric matrix. This can be seen by differentiating the relation that ensures $R$ is orthogonal

$$
\frac{d}{d \theta} R R^{T}=\frac{d R}{d \theta} R^{T}+R \frac{d R^{T}}{d \theta}=\frac{d I}{d \theta}=0
$$

when $\theta=0$ we have that $R=I$ and hence,

$$
\frac{d R(0)}{d \theta}+\frac{d R(0)^{T}}{d \theta}=0
$$

Now a $3 \times 3$ anti-symmetric matrix corresponds to a 3 -dimensional vector,

$$
\Omega=\left(\begin{array}{ccc}
0 & -\omega_{z} & \omega_{y} \\
\omega_{z} & 0 & -\omega_{x} \\
-\omega_{y} & \omega_{x} & 0
\end{array}\right)
$$

where $\omega_{i}$ are the components of the vector, $\boldsymbol{\omega}$. With these definitions the product of the matrix $\Omega$ with any 3 -dimensional vector exactly models the vector product with $\boldsymbol{\omega}$, that is, $\Omega \mathbf{p}=\boldsymbol{\omega} \times \mathbf{p}$ for any $\mathbf{p}$.

Hence, we can see that $\boldsymbol{\omega}$ is the angular velocity vector of the body and $\omega$ is its magnitude.

A general element of the Lie algebra $s e(3)$, is a matrix with the partitioned form,

$$
S=\left(\begin{array}{cc}
\Omega & \mathbf{v} \\
0 & 0
\end{array}\right)
$$

These matrices form a 6-dimensional vector space and it is often useful to write the elements of the space as six-dimensional vectors,

$$
\mathbf{s}=\left(\begin{array}{l}
\omega \\
\mathbf{v}
\end{array}\right)
$$

\section{The Adjoint Representation}

So far the group $S E(3)$ has been defined by it $4 \times 4$ matrix representation. Sometimes it is useful to think of the group as an abstract object and consider different representations of it. A representation here 
is a linear representation, that is, we represent group elements by matrices, or more genrerally linear transformations. The group product is modeled by matrix multiplication and inverses in the group by matrix inversion. For any group there are many such representations, here we look at a representation which can be defined for any group. Any Lie group acts linearly on its Lie algebra. This representation is known as the adjoint representation of the group.

For $S E(3)$ we can write this in terms of the $4 \times 4$ matrices as

$$
S^{\prime}=A S A^{-1}
$$

where $A=A(g)$ is the $4 \times 4$ matrix corresponding to some group element $g$. In terms of the 6-dimensional vectors representing the Lie algebra, we get,

$$
\mathbf{s}^{\prime}=\operatorname{Ad}(g) \mathbf{s}
$$

Here $\operatorname{Ad}(g)$ is a $6 \times 6$ matrix representing the group element $g$. The general form of the matrices in this adjoint representation is,

$$
\operatorname{Ad}(g)=\left(\begin{array}{cc}
R & 0 \\
T R & R
\end{array}\right)
$$

again $R$ is a $3 \times 3$ rotation matrix but $T$ is a $3 \times 3$ anti-symmetric matrix corresponding to a translation vector $\mathbf{t}$.

This adjoint action of the group on its Lie algebra can be extended to an action of the Lie algebra on itself. In terms of the $4 \times 4$ representation of $S E(3)$ this can be explained as follows. Take $S_{2}$ to be an element of the Lie algebra, as a $4 \times 4$ matrix. Now suppose $A(\theta)$ is a oneparameter subgroups corresponding to a Lie algebra element $S_{1}$, that is $d A(0) / d \theta=S_{1}$. Now if we differentiate the adjoint action of the group, $A S_{2} A^{-1}$ and set $\theta=0$ we get the commutator,

$$
S_{1} S_{2}-S_{2} S_{1}=\left[S_{1}, S_{2}\right]
$$

In terms of 6-dimensional vectors the commutator can be written as

$$
\operatorname{ad}\left(\mathbf{s}_{1}\right) \mathbf{s}_{2}=\left(\begin{array}{cc}
\Omega_{1} & 0 \\
V_{1} & \Omega_{1}
\end{array}\right)\left(\begin{array}{l}
\boldsymbol{\omega}_{2} \\
\mathbf{v}_{2}
\end{array}\right)
$$

The notation ad, lower case, denotes the adjoint action of the Lie algebra on itself. The commutators of elements are a key feature of Lie algebras, in robotics these commutators turn up in the dynamics of robots and in many other applications. Notice that in the 6-dimensional, partitioned form of representation of the Lie algebra, commutators can be written,

$$
\left[\mathbf{s}_{1}, \mathbf{s}_{2}\right]=\operatorname{ad}\left(\mathbf{s}_{1}\right) \mathbf{s}_{2}=\left(\begin{array}{c}
\boldsymbol{\omega}_{1} \times \boldsymbol{\omega}_{2} \\
\mathbf{v}_{1} \times \boldsymbol{\omega}_{2}+\boldsymbol{\omega}_{1} \times \mathbf{v}_{2}
\end{array}\right)
$$




\section{The Exponential Map}

The exponential map sends Lie algebra elements to the Lie group. In a region of the origin the map is guaranteed to be a homeomorphism, and hence we can use the Lie algebra as a coordinate system for the group but only in a patch containing the identity. For $S E(3)$, this patch is quite big, we just have to keep rotation angles to between $\pm \pi$. The exponential map provides the link between the Lie algebra and the group.

In general, the exponential of a matrix $X$ is defined by the series,

$$
e^{X}=I+X+\frac{1}{2 !} X^{2}+\frac{1}{3 !} X^{3}+\cdots
$$

where $X^{2}$ is the matrix product $X^{2}=X X$ etc. It is possible to show that the series converges and, if $X$ is a matrix representing an element of a Lie algebra, then the exponential $e^{X}$ will be a matrix representing an element of the corresponding Lie group. The mapping is essentially independent of the representation.

The product of a pair of matrix exponentials is generally not simply the exponential of the sum of the exponents. This is because the exponents may not commute. However, if $S$ is a fixed screw, then it is clear that

$$
e^{\theta_{1} S} e^{\theta_{2} S}=e^{\left(\theta_{1}+\theta_{2}\right) S}
$$

So these are the 1-parameter subgroups again, and we can write,

$$
A(\theta)=e^{\theta S}
$$

This means that we can associate a screw $S$, to any 1-degree-of-freedom mechanical joint. We will see how to do this in detail in a moment. For now simply observe that the forward kinematics for a six-joint industrial robot, as outlined in section 6 above, can be written as

$$
K(\boldsymbol{\theta})=e^{\theta_{1} S_{1}} e^{\theta_{2} S_{2}} e^{\theta_{3} S_{3}} e^{\theta_{4} S_{4}} e^{\theta_{5} S_{5}} e^{\theta_{6} S_{6}}
$$

The parameters $\theta_{i}$, are usually referred to as the joint parameters, or joint angles if the joints are revolute. This form of forward kinematics of serial robots was introduced by Brockett [2].

As promised, we next look at how to associate a screw to a mechanical joint. To each joint we can associate a 1-parameter subgroup,

$$
e^{\theta S}=\left(\begin{array}{cc}
R & \frac{\theta p}{2 \pi} \hat{\mathbf{x}}+(I-R) \mathbf{u} \\
0 & 1
\end{array}\right)
$$

where the screw $S$ has the form,

$$
S=\left(\begin{array}{cc}
\Omega & \mathbf{v} \\
0 & 0
\end{array}\right)
$$


If we look at the rotation part first we expect that $\hat{\mathbf{x}}$ to be along the axis of rotation so that $R \hat{\mathbf{x}}=\hat{\mathbf{x}}$. Differentiating this with respect to $\theta$ and setting $\theta=0$ we get,

$$
\Omega \hat{\mathbf{x}}=\boldsymbol{\omega} \times \hat{\mathbf{x}}=0
$$

From this we can conclude that $\boldsymbol{\omega}$ is proportional to $\hat{\mathbf{x}}$. In fact, it makes sense to define $\boldsymbol{\omega}=\hat{\mathbf{x}}$ so that the parameter $\theta$, becomes the rotation angle. Next, we look at $\mathbf{u}$, a point on the axis of the screw motion. Here we expect,

$$
A(\theta)\left(\begin{array}{c}
\mathbf{u} \\
1
\end{array}\right)=\left(\begin{array}{c}
\mathbf{u}+\frac{\theta p}{2 \pi} \hat{\mathbf{x}} \\
1
\end{array}\right)
$$

that is, a point on the axis of the screw is simply translated alog the axis. The derivative at $\theta=0$ gives

$$
S\left(\begin{array}{c}
\mathbf{u} \\
1
\end{array}\right)=\left(\begin{array}{c}
\frac{p}{2 \pi} \hat{\mathbf{x}} \\
0
\end{array}\right)
$$

That is,

$$
\Omega \mathbf{u}+\mathbf{v}=\frac{\theta p}{2 \pi} \hat{\mathbf{x}}
$$

Replacing $\hat{\mathbf{x}}$ by $\boldsymbol{\omega}$ and rearranging we get the result

$$
\mathbf{v}=\mathbf{u} \times \boldsymbol{\omega}+\frac{p}{2 \pi} \boldsymbol{\omega}
$$

Notice that if the pitch of the joint vanishes $p=0$, that is the joint is revolute, then the screw we associate with it,

$$
\mathbf{s}=\left(\begin{array}{c}
\omega \\
\mathbf{u} \times \omega
\end{array}\right)
$$

is simply given by the Plücker coordinates for the line forming the axis of the joint. For helical joints we add the extra term $\frac{p}{2 \pi} \boldsymbol{\omega}$ to the $\mathbf{v}$ part of the screw, where $p$ is the pitch of the joint. The above does not apply to prismatic joints, but they are easy to deal with. Using the same sort of argument as above, a prismatic joint which allows movement in the direction $\hat{\mathbf{v}}$ will be represented by a screw,

$$
\mathbf{s}=\left(\begin{array}{l}
\mathbf{0} \\
\hat{\mathbf{v}}
\end{array}\right)
$$

In general it is quite difficult to compute matrix exponentials, but in the case of rigid body motions we are lucky, the problem is relatively straightforward. 
Consider a typical element from the $4 \times 4$ representation,

$$
S=\left(\begin{array}{cc}
\Omega & \mathbf{v} \\
0 & 0
\end{array}\right)
$$

Any such matrix satisfies the polynomial equation,

$$
S^{4}+|\omega|^{2} S^{2}=0
$$

this can be checked by direct computation. The equation factorises as

$$
S^{4}+|\omega|^{2} S^{2}=S^{2}(S+i|\omega| I)(S-i|\omega| I)
$$

Now we can find three matrix functions of $S$, labelled $P_{0}, P_{+}$and $P_{-}$, which satisfy $P_{i}^{2}=P_{i}$ and $P_{i} P_{j}=0$ if $i \neq j$, such a system is called a system of mutually annihilating idempotents. These matrices are given by,

$$
\begin{aligned}
P_{0} & =\frac{1}{|\omega|^{2}}\left(S^{2}+|\omega|^{2} I\right), \\
P_{+} & =\frac{1}{2 i|\omega|^{3}} S^{2}(S-i|\omega| I), \\
P_{-} & =\frac{-1}{2 i|\omega|^{3}} S^{2}(S+i|\omega| I) .
\end{aligned}
$$

here $i=\sqrt{-1}$ is the imaginary unit. We also require a nilpotent here, $N_{0}=\frac{1}{|\omega|^{2}} S\left(S^{2}+|\omega|^{2} I\right)$. This nilpotent satisfies $N_{0}^{2}=0, N_{0} P_{+}=$ $N_{0} P_{-}=0$ and $N_{0} P_{0}=N_{0}$.

The point of all this is that we can write our original matrix $S$ as

$$
S=N_{0}-i|\omega| P_{+}+i|\omega| P_{-}
$$

and so when we take powers of $S$ we only need to take powers of the coefficients of $P_{+}$and $P_{-}$since there are no cross-terms and the powers of the matrices are simple. So exponentiating we get,

$$
e^{S}=I+N_{0}+\left(e^{-i|\omega|}-1\right) P_{+}+\left(e^{i|\omega|}-1\right) P_{-}
$$

It is also straightforward to see that $I=P_{0}+P_{+}+P_{-}$so the exponential can be simplified to,

$$
e^{S}=P_{0}+N_{0}+e^{-i|\omega|} P_{+}+e^{i|\omega|} P_{-}
$$

Finally, substituting back the $S$ s and replacing the complex exponentials with trigonometric functions we have the result

$$
e^{S}=I+S+\frac{1}{|\omega|^{2}}(1-\cos |\omega|) S^{2}+\frac{1}{|\omega|^{3}}(|\omega|-\sin |\omega|) S^{3}
$$


The corresponding formula for the group $S O(3)$ is known as the Rodrigues formula.

We can produce a similar result for the adjoint representation of $S E(3)$. A typical element of the Lie algebra in this representation is given by,

$$
\operatorname{ad}(\mathbf{s})=\left(\begin{array}{ll}
\Omega & 0 \\
V & \Omega
\end{array}\right)
$$

In general we would expect a $6 \times 6$ matrix to satisfy a degree 6 polynomial equation, these matrices however, satisfy a degree 5 equation,

$$
\operatorname{ad}(\mathbf{s})^{5}+2|\omega|^{2} \operatorname{ad}(\mathbf{s})^{3}+|\omega|^{4} \operatorname{ad}(\mathbf{s})=0
$$

This relation can be verified easily using the following relation between pairs of $3 \times 3$ anti-symmetric matrices,

$$
\Omega^{2} V \Omega^{2}+|\omega|^{2}(\Omega V+V \Omega)=-|\omega|^{4} V
$$

In turn this relation can be verified by using the connection between these matrices and vector products, $V \mathbf{x}=\mathbf{v} \times \mathbf{x}$ for any vector $\mathbf{x}$, and then using the familiar formula for vector triple products.

The degree 5 equation above factorises as

$$
\operatorname{ad}(\mathbf{s})^{5}+2|\omega|^{2} \operatorname{ad}(\mathbf{s})^{3}+|\omega|^{4} \operatorname{ad}(\mathbf{s})=\operatorname{ad}(\mathbf{s})(\operatorname{ad}(\mathbf{s})+i|\omega| I)^{2}(\operatorname{ad}(\mathbf{s})-i|\omega| I)^{2}
$$

So this time we have two repeated factors and hence we seek three idempotents as before and two nilpotents, one associated with each repeated factor.

$$
\begin{aligned}
P_{0} & =\frac{1}{|\omega|^{4}}(\operatorname{ad}(\mathbf{s})+i|\omega| I)^{2}(\operatorname{ad}(\mathbf{s})-i|\omega| I)^{2} \\
& =\left(\operatorname{ad}(\mathbf{s})^{4}+2|\omega|^{2} \operatorname{ad}(\mathbf{s})^{2}+|\omega|^{4} I\right) /|\omega|^{4} \\
P_{+} & =\frac{-1}{4|\omega|^{4}} \operatorname{ad}(\mathbf{s})(\operatorname{ad}(\mathbf{s})-i|\omega| I)^{2}(2 \operatorname{ad}(\mathbf{s})+3 i|\omega| I) \\
& =-\left(2 \operatorname{ad}(\mathbf{s})^{4}-i|\omega| \operatorname{ad}(\mathbf{s})^{3}+4|\omega|^{2} \operatorname{ad}(\mathbf{s})^{2}-3 i|\omega|^{3} \operatorname{ad}(\mathbf{s})\right) / 4|\omega|^{4} \\
N_{+} & =\frac{1}{4|\omega|^{4}} \operatorname{ad}(\mathbf{s})(\operatorname{ad}(\mathbf{s})+i|\omega| I)(\operatorname{ad}(\mathbf{s})-i|\omega| I)^{2} \\
& =\left(\operatorname{ad}(\mathbf{s})^{4}-i|\omega| \operatorname{ad}(\mathbf{s})^{3}+|\omega|^{2} \operatorname{ad}(\mathbf{s})^{2}-i|\omega|^{3} \operatorname{ad}(\mathbf{s})\right) / 4|\omega|^{4} \\
P_{-} & =\frac{-1}{4|\omega|^{4}} \operatorname{ad}(\mathbf{s})(\operatorname{ad}(\mathbf{s})+i|\omega| I)^{2}(2 \operatorname{ad}(\mathbf{s})-3 i|\omega| I) \\
& =-\left(2 \operatorname{ad}(\mathbf{s})^{4}+i|\omega| \operatorname{ad}(\mathbf{s})^{3}+4|\omega|^{2} \operatorname{ad}(\mathbf{s})^{2}+3 i|\omega|^{3} \operatorname{ad}(\mathbf{s})\right) / 4|\omega|^{4} \\
N_{-} & =\frac{1}{4|\omega|^{4}} \operatorname{ad}(\mathbf{s})(\operatorname{ad}(\mathbf{s})+i|\omega| I)^{2}(\operatorname{ad}(\mathbf{s})-i|\omega| I) \\
& =\left(\operatorname{ad}(\mathbf{s})^{4}+i|\omega| \operatorname{ad}(\mathbf{s})^{3}+|\omega|^{2} \operatorname{ad}(\mathbf{s})^{2}+I|\omega|^{3} \operatorname{ad}(\mathbf{s})\right) / 4|\omega|^{4}
\end{aligned}
$$


By inspection we have that

$$
\operatorname{ad}(\mathbf{s})=-i|\omega| P_{+}-i|\omega| N_{+}+i|\omega| P_{-}+i|\omega| N_{-}
$$

and as usual we also have,

$$
I=P_{0}+P_{+}+P_{-}
$$

Notice that with matrices $P$ and $N$ such that $P^{2}=P, N^{2}=0$ and $P N=N$ the $k$ th power of their sum is simply, $(P+N)^{k}=P+k N$. Hence the exponential of a matrix ad(s) from the adjoint representation of $s e(3)$ can be written as

$$
e^{\operatorname{ad}(\mathbf{s})}=I+\left(e^{-i|\omega|}-1\right) P_{+}-i|\omega| e^{-i|\omega|} N_{+}+\left(e^{i|\omega|}-1\right) P_{-}+i|\omega| e^{i|\omega|} N_{-}
$$

or

$$
e^{\operatorname{ad}(\mathbf{s})}=P_{0}+e^{-i|\omega|} P_{+}-i|\omega| e^{-i|\omega|} N_{+}+e^{i|\omega|} P_{-}+i|\omega| e^{i|\omega|} N_{-}
$$

Expanding the idempotents and nilpotents in terms of powers of ad(s) we finally obtain the result

$$
\begin{aligned}
e^{\operatorname{ad}(\mathbf{s})=I} & +\frac{1}{2|\omega|}(3 \sin |\omega|-|\omega| \cos |\omega|) \operatorname{ad}(\mathbf{s}) \\
& +\frac{1}{2|\omega|^{2}}(4-4 \cos |\omega|-|\omega| \sin |\omega|) \operatorname{ad}(\mathbf{s})^{2} \\
& +\frac{1}{2|\omega|^{3}}(\sin |\omega|-|\omega| \cos |\omega|) \operatorname{ad}(\mathbf{s})^{3} \\
& +\frac{1}{2|\omega|^{4}}(2-2 \cos |\omega|-|\omega| \sin |\omega|) \operatorname{ad}(\mathbf{s})^{4}
\end{aligned}
$$

\section{Derivatives of Exponentials}

Suppose we are given a path in a Lie group $(S E(3)$ here) as an exponential,

$$
g(t)=e^{X(t)} .
$$

How can we find the derivative of this formula and what does it represent?

In the previous section we met the simple case where the path in the Lie algebra $X(t)$ is just $t X_{0}$ where $X_{0}$ is a constant element in the algebra. The derivative of such a path is simply,

$$
\frac{d}{d t} e^{t X_{0}}=X_{0} e^{t X_{0}}
$$


However, when $X(t)$ is more complicated this is no longer valid. The problem is that $d X / d t=\dot{X}$ does not necessarily commute with $X$ so differentiating the expansion of the exponential gives

$$
\begin{aligned}
\frac{d}{d t} e^{X}= & \dot{X}+\frac{1}{2 !}(\dot{X} X+X \dot{X})+\frac{1}{3 !}\left(\dot{X} X^{2}+X \dot{X} X+X^{2} \dot{X}\right)+\cdots \\
& \cdots+\frac{1}{(k+1) !}\left(\dot{X} X^{k}+X \dot{X} X^{k-1}+\cdots+X^{k} \dot{X}\right)+\cdots
\end{aligned}
$$

In [3] Hausdorff showed that

$$
\left(\frac{d}{d t} e^{X}\right) e^{-X}=\dot{X}+\frac{1}{2 !}[X, \dot{X}]+\frac{1}{3 !}[X,[X, \dot{X}]]+\frac{1}{4 !}[X,[X,[X, \dot{X}]]]+\cdots
$$

The right-hand side of this equation will be abbreviated to $X_{d}$. Notice that this matrix is composed of sums of iterated commutators and hence $X_{d}$ is an element of the Lie algebra. The Hausdorff formula above implies that

$$
\frac{d}{d t} e^{X}=X_{d} e^{X}
$$

In the rotation group $S O(3)$ this means that $X_{d}$ corresponds to the angular velocity of the motion. In $S E(3)$, the group of rigid body motions the corresponding vector is the velocity screw of the motion. The equation is important in several applications. In a typical dynamical simulation, for example, equations of motion would be solved numerically to find the velocity screw or, angular and linear velocities. From these quantities the transformation matrices usually have to be found. It is essentially the above equation that has to be solved to find the rigid transformation undergone by the rigid body under consideration. In most commercial applications a rudimentary approach to this problem is often taken. This is because the speed of computation is far more important than the accuracy.

Using vectors to represent the Lie algebra the Hausdorff formula can be written neatly as

$$
\mathbf{x}_{d}=\sum_{k=0}^{\infty} \frac{1}{(k+1) !} \operatorname{ad}^{k}(\mathbf{x}) \dot{\mathbf{x}}
$$

with $\operatorname{ad}()$ the adjoint representation of the Lie algebra, as usual. 
For $S E(3)$ we can evaluate this infinite sum using the idempotents and nilpotents found in section 9 above. This gives

$$
\begin{aligned}
\sum_{k=0}^{\infty} \frac{1}{(k+1) !} \operatorname{ad}(\mathbf{s})^{k}= & P_{0}+\sum_{k=0}^{\infty} \frac{(-i|\omega|)^{k}}{(k+1) !} P_{+}+\sum_{k=0}^{\infty} \frac{k(-i|\omega|)^{k}}{(k+1) !} N_{+} \\
& +\sum_{k=0}^{\infty} \frac{(i|\omega|)^{k}}{(k+1) !} P_{-}+\sum_{k=0}^{\infty} \frac{k(i|\omega|)^{k}}{(k+1) !} N_{-}
\end{aligned}
$$

Evaluating the infinite sums gives

$$
\begin{aligned}
\sum_{k=0}^{\infty} \frac{1}{(k+1) !} \operatorname{ad}(\mathbf{s})^{k}=P_{0} & +\frac{1}{-i|\omega|}\left(e^{-i|\omega|}-1\right) P_{+} \\
& +\frac{1}{-i|\omega|}\left((-i|\omega|-1) e^{-i|\omega|}+1\right) N_{+} \\
& +\frac{1}{i|\omega|}\left(e^{i|\omega|}-1\right) P_{-} \\
& +\frac{1}{i|\omega|}\left((i|\omega|-1) e^{i|\omega|}+1\right) N_{-}
\end{aligned}
$$

Substituting for the idemponents and nilpotents gives

$$
\begin{aligned}
\sum_{k=0}^{\infty} \frac{1}{(k+1) !} \operatorname{ad}(\mathbf{s})^{k}=I & +\frac{1}{2|\omega|^{2}}(4-|\omega| \sin |\omega|-4 \cos |\omega|) \operatorname{ad}(\mathbf{s}) \\
& +\frac{1}{2|\omega|^{3}}(4|\omega|-5 \sin |\omega|+|\omega| \cos |\omega|) \operatorname{ad}(\mathbf{s})^{2} \\
& +\frac{1}{2|\omega|^{4}}(2-|\omega| \sin |\omega|-2 \cos |\omega|) \operatorname{ad}(\mathbf{s})^{3} \\
& +\frac{1}{2|\omega|^{5}}(2|\omega|-3 \sin |\omega|+|\omega| \cos |\omega|) \operatorname{ad}(\mathbf{s})^{4}
\end{aligned}
$$

This format is not particularly useful for dynamic simulation, it would be more useful to have $\dot{\mathbf{s}}$ as the subject of the equation. This would allow direct numerical methods to be used. Such an inversion is possible in general, see [3]. The result is another infinite series, however it is not necessary to follow this route since the above formula can be inverted more directly using the idempotents. Recall that $P_{0}+P_{+}+P_{-}=I$ so that for any constants $a_{i}, b_{i}$,

$$
\begin{aligned}
\left(a_{0} P_{0}+a_{+} P_{+}+b_{+} N_{+}+a_{-} P_{-}+b_{-} N_{-}\right) \\
\\
\quad *\left(\frac{1}{a_{0}} P_{0}+\frac{1}{a_{+}} P_{+}-\frac{b_{+}}{a_{+}^{2}} N_{+}+\frac{1}{a_{-}} P_{-}-\frac{b_{-}}{a_{-}^{2}} N_{-}\right) \\
=\left(P_{0}+P_{+}+P_{-}\right)=I
\end{aligned}
$$


The computations are a little more than can be comfortably done by hand, but are readily computed using a computer algebra package such as Maple or Mathematica.

Let, $\mathbf{s}_{d}$ be the Lie algebra element satisfying $\frac{d}{d t} e^{\operatorname{ad}(\mathbf{s})}=\operatorname{ad}\left(\mathbf{s}_{d}\right) e^{\operatorname{ad}(\mathbf{s})}$, then we have the result

$$
\begin{aligned}
\dot{\mathbf{s}}=(I & -\frac{1}{2} \operatorname{ad}(\mathbf{s})+\left(\frac{2}{|\omega|^{2}} \frac{|\omega|+3 \sin |\omega|}{4|\omega|(\cos |\omega|-1)}\right) \operatorname{ad}(\mathbf{s})^{2}+ \\
& \left.+\left(\frac{1}{|\omega|^{4}}+\frac{|\omega|+\sin |\omega|}{4|\omega|^{3}(\cos |\omega|-1)}\right) \operatorname{ad}(\mathbf{s})^{4}\right) \mathbf{s}_{d}
\end{aligned}
$$

Notice the absence of a term in $\operatorname{ad}(\mathbf{s})^{3}$ in the above.

Finally here, we look at the interpretation of these derivatives. Consider a general sequence of rigid motions parameterised by time $t$, then we can see from the above that

$$
\frac{d A}{d t} A^{-1}=S_{d}
$$

is an element of the Lie algebra and that it corresponds to the instantaneous velocity. The $\Omega$ or $\boldsymbol{\omega}$ part is the angular velocity while the $\mathbf{v}$ part is the linear velocity of a point on the screw axis. We can find the velocity of a point on the body moving with this motion as follows. At time $t=0$ the point will be assumed to have position vector $\mathbf{p}_{0}$, at any subsequent time its position will be given by,

$$
\left(\begin{array}{c}
\mathbf{p} \\
1
\end{array}\right)=A(t)\left(\begin{array}{c}
\mathbf{p}_{0} \\
1
\end{array}\right)
$$

Differentiating with respect to time gives

$$
\left(\begin{array}{c}
\dot{\mathbf{p}} \\
0
\end{array}\right)=\dot{A} A^{-1}\left(\begin{array}{l}
\mathbf{p} \\
1
\end{array}\right)=S_{d}\left(\begin{array}{l}
\mathbf{p} \\
1
\end{array}\right)
$$

Hence the velocity of the point is,

$$
\dot{\mathbf{p}}=\boldsymbol{\omega} \times \mathbf{p}+\mathbf{v}
$$

The screw $S_{d}$ or $\mathbf{s}_{d}$ will be called the velocity screw of the motion.

\section{Jacobians}

The exponential form of robot kinematics is useful for deriving differential properties, for example Jacobians.

Consider a point attached to the end-effector of the robot, in the robot's home position the point has position vector $\mathbf{p}$. Hence, at any 
subsequent configuration of the robot the position of the point will be given by,

$$
\left(\begin{array}{c}
\mathbf{p}^{\prime} \\
1
\end{array}\right)=K(\boldsymbol{\theta})\left(\begin{array}{l}
\mathbf{p} \\
1
\end{array}\right)
$$

If the joints are moving with rates, $\dot{\theta}_{1}, \dot{\theta}_{2}, \ldots \dot{\theta}_{6}$ then the velocity of the point will be,

$$
\left(\begin{array}{c}
\dot{\mathbf{p}} \\
0
\end{array}\right)=\left(\dot{\theta}_{1} \frac{\partial K}{\partial \theta_{1}}+\dot{\theta}_{2} \frac{\partial K}{\partial \theta_{2}}+\cdots+\dot{\theta}_{6} \frac{\partial K}{\partial \theta_{6}}\right)\left(\begin{array}{c}
\mathbf{p} \\
1
\end{array}\right)
$$

The partial derivatives are easy to evaluate at the home position,

$$
\frac{\partial K}{\partial \theta_{i}}=e^{\theta_{1} S_{1}} \cdots S_{i} e^{\theta_{i} S_{i}} \cdots e^{\theta_{6} S_{6}}=S_{i}
$$

So in the home position the velocity of the point is,

$$
\left(\begin{array}{c}
\dot{\mathbf{p}} \\
0
\end{array}\right)=\left(\dot{\theta}_{1} S_{1}+\dot{\theta}_{2} S_{2}+\cdots+\dot{\theta}_{6} S_{6}\right)\left(\begin{array}{c}
\mathbf{p} \\
1
\end{array}\right)
$$

This is more general than it looks. If we choose the current configuration of the robot to be the home configuration then the above argument will apply. So, $S_{i}$ here will be taken to mean the current position of the $i$-th joint. Alternatively, we could let $\mathbf{s}_{i}^{0}$ denote the home position of the robot's joint screws. Subsequent configurations for the joints will be given by,

$$
\mathbf{s}_{i}=e^{\theta_{1} \operatorname{ad}\left(\mathbf{s}_{1}^{0}\right)} e^{\theta_{2} \operatorname{ad}\left(\mathbf{s}_{2}^{0}\right)} \cdots e^{\theta_{i-1} \operatorname{ad}\left(\mathbf{s}_{i-1}^{0}\right)} \mathbf{s}_{i}^{0}
$$

Hence, we have that

$$
\frac{\partial K}{\partial \theta_{i}} K^{-1}=S_{i}
$$

So we can find the velocity screw of the robot's end-effector by simply substituting the adjoint representation of the Lie algebra. Hence,

$$
\left(\begin{array}{l}
\boldsymbol{\omega} \\
\mathbf{v}
\end{array}\right)=\mathbf{s}_{1} \dot{\theta}_{1}+\mathbf{s}_{2} \dot{\theta}_{2}+\cdots+\mathbf{s}_{6} \dot{\theta}_{6}
$$

If we compare this with the Jacobian relation for the velocity $\mathbf{s}_{d}=J \dot{\boldsymbol{\theta}}$, we can see that the columns of the $6 \times 6$ Jacobian matrix must be the current joint screws of the robot,

$$
J=\left(\mathbf{s}_{1}\left|\mathbf{s}_{2}\right| \mathbf{s}_{3}\left|\mathbf{s}_{4}\right| \mathbf{s}_{5} \mid \mathbf{s}_{6}\right)
$$

A well known result. 
An immediate application of the above is to robot control. Suppose we wanted the robots end-effector to follow a path given by a exponential, $e^{\mathbf{s}(t)}$. In a traditional approach to this problem, the rigid motions along the path would be computed and then a inverse kinematic routine would be used to find the corresponding joint angles. It is probably more efficient to find the joint angles by numerically integrating the equation,

$$
J \dot{\boldsymbol{\theta}}=\mathbf{s}_{d}
$$

For most current designs of industrial the Jacobian matrix $J$ can be inverted symbolically, although this doesn't seem to be widely appreciated. Also $\mathbf{s}_{d}$ can be computed using the formulas given above.

\section{Concluding Remarks}

In this brief lecture there has only been enough time to give an outline of how group theory can be used in robotics. Several important applications have been omitted in the interests of brevity. Although we have discussed the forward kinematics of serial manipulators the inverse kinematics is an key problem in the subject. Here we know where we want to place the robot's end-effector and we must compute the joint angles needed to achieve this. Although, an approach using differential equations has been sketched above, this clearly runs into difficulties when the robot's Jacobian becomes singular. The investigation of these robot singularities is another problem where group theory has been usefully applied, see [6]. For parallel mechanism, such as the Gough-Stewart platform, the inverse kinematics are straightforward, it is the forward kinematics which are hard.

Robot dynamics requires another couple of representation of the group $S E(3)$. First, the forces and torques acting on a rigid body can be combined into a single 6 -dimensional vector called a wrench. The wrenches transform according to a representation dual to the adjoint representation, the coadjoint representation. An accidental property of $S E(3)$ means that the adjoint and coadjoint representations are similar. In Ball's original screw theory velocities and wrenches were treated as the same sort of object, however, to make progress it is simplest to separate

them. The second representation we need is the symmetric product of the adjoint representation. Elements of this representation can then be interpreted as $6 \times 6$ inertia matrices.

There are many applications of group theory to robot vision. Now the group may be different. For example, there are formulations of the camera calibration problem that use the group $S L(4)$ to model the possible camera parameters. Certainly projective geometry will be important when discussing cameras and there are projective groups associated with 
this geometry. The group relevant to geometric optics is the symplectic group $S p(6, \mathbb{R})$, this is the symmetry groups of a complex of lines in space.

Finally, the above presentation has used matrices and vectors exclusively. There is some merit to using Clifford algebras to represent the group of rigid body motions and the various spaces on which it acts. Relations and formulas in Clifford algebra tend to be neater than their matrix counterparts and this is useful for symbolic and hand computation. Another advantage of the Clifford algebra is that the group $S E(3)$ can be represented very compactly and this allows us to study the geometry of the group itself. See Joan Lasenby's lecture later in this meeting.

\section{References}

[1] R.S. Ball. The Theory of Screws. Cambridge University Press, Cambridge, 1900.

[2] R. Brockett. Robotic manipulators and the product of exponential formula. In P. Fuhrman ed. Proc. Mathematical Theory of Networks and Systems pp. 120-129, 1984.

[3] F. Hausdorff. Die Symbolische exponential formel in den gruppen theorie. Berichte de Sächicen Akademie de Wissenschaften (Math Phys Klasse) vol. 58, pp. 19-48, 1906.

[4] J.M. Hervé Analyse Structurelle de Mecanismes par Groupe des Displacements. Mechanism and Machine Theory 13:437-450, 1978.

[5] B.K.P Horn. Robot Vision Cambridge, MA:MIT Press, 1986. 1987.

[6] A. Karger. Classification of serial robot-manipulators with non-removable singularities. J. Mech. Design 30:202-208, 1996.

[7] F. Reuleaux Theoretische Kinematic: Grunzüge einer Theorie des Maschinwesens Braunschweig: Vieweg, 1875. Trans. A.B. W. Kennedy as The Kinematics of Machinery, London: Macmillan, 1876. Reprinted, New York: Dover, 1963. 